\title{
Chemoproteomics reveals Toll-like receptor fatty acylation
}

\author{
Nicholas M Chesarino ${ }^{1}$, Jocelyn C Hach ${ }^{1}$, James L Chen², Balyn W Zaro ${ }^{3}$, Murugesan VS Rajaram', Joanne Turner', \\ Larry S Schlesinger ${ }^{1}$, Matthew R Pratt ${ }^{3}$, Howard C Hang $^{4}$ and Jacob S Yount ${ }^{1^{*}}$
}

\begin{abstract}
Background: Palmitoylation is a 16-carbon lipid post-translational modification that increases protein hydrophobicity. This form of protein fatty acylation is emerging as a critical regulatory modification for multiple aspects of cellular interactions and signaling. Despite recent advances in the development of chemical tools for the rapid identification and visualization of palmitoylated proteins, the palmitoyl proteome has not been fully defined. Here we sought to identify and compare the palmitoylated proteins in murine fibroblasts and dendritic cells.

Results: A total of 563 putative palmitoylation substrates were identified, more than 200 of which have not been previously suggested to be palmitoylated in past proteomic studies. Here we validate the palmitoylation of several new proteins including Toll-like receptors (TLRs) 2, 5 and 10, CD80, CD86, and NEDD4. Palmitoylation of TLR2, which was uniquely identified in dendritic cells, was mapped to a transmembrane domain-proximal cysteine. Inhibition of TLR2 S-palmitoylation pharmacologically or by cysteine mutagenesis led to decreased cell surface expression and a decreased inflammatory response to microbial ligands.

Conclusions: This work identifies many fatty acylated proteins involved in fundamental cellular processes as well as cell type-specific functions, highlighting the value of examining the palmitoyl proteomes of multiple cell types. Spalmitoylation of TLR2 is a previously unknown immunoregulatory mechanism that represents an entirely novel avenue for modulation of TLR2 inflammatory activity.
\end{abstract}

Keywords: Palmitoylation, Post-translational modification, Click chemistry, Toll-like receptor, TLR2, Fatty acylation, Proteomics

\section{Background}

Protein palmitoylation is the addition of a 16-carbon fatty acid primarily to cysteines via a thioester linkage (termed S-palmitoylation) [1]. This form of fatty acylation targets cytoplasmic proteins to membranes, but can also occur on transmembrane proteins where it often affects protein localization or stability [1-3]. Identification and characterization of palmitoylated proteins has traditionally been encumbered because of insensitive detection methods, the lack of a consensus amino acid motif for bioinformatics prediction of palmitoylation sites, a lack of antibodies for detecting palmitoylation and challenges in detecting palmitoylated peptides with standard mass spectrometry techniques $[4,5]$. To

\footnotetext{
* Correspondence: yount.37@osu.edu

${ }^{1}$ Department of Microbial Infection and Immunity, Center for Microbial Interface Biology, The Ohio State University, Columbus, OH 43210, USA Full list of author information is available at the end of the article
}

overcome these difficulties, non-radioactive chemical tools and methodologies have been recently developed that are advancing the study of protein palmitoylation [6-11]. An analogue of palmitic acid possessing a terminal alkyne group (alk-16, Figure 1A) is incorporated by cells onto proteins at sites of palmitoylation [6]. The alkynyl group allows a targeted reaction with azidefunctionalized detection tags via the copper-catalyzed azide-alkyne cycloaddition reaction commonly termed click chemistry (Figure 1A). Azido-rhodamine (az-rho) is a detection tag that can be used for visualization, while azido-azo-biotin (az-biotin) allows selective retrieval of alk-16-labeled proteins using streptavidincoated agarose $[7,8,12]$. We, and others, have previously used, and continue to apply these tools for enhancing our understanding of regulation of immune responses by lipid post-translational modifications (PTMs) [7,11,13-17]. 


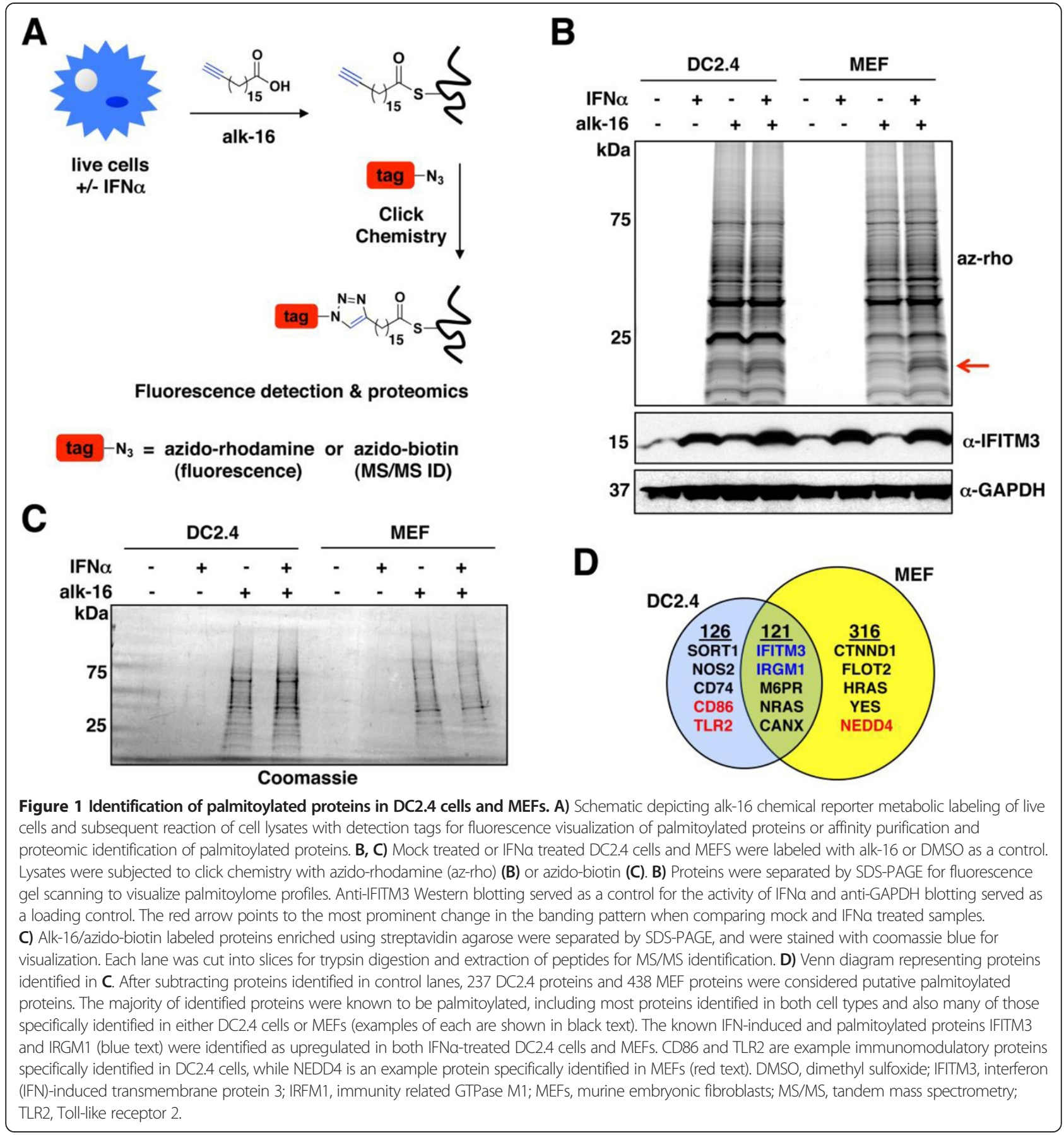

Of particular importance, the aforementioned chemical tools allowed the identification of palmitoylation-dependent antiviral activity of the interferon (IFN)-induced transmembrane protein 3 (IFITM3) [7]. In the present study, we performed an analysis of palmitoylated proteins in a murine antigen presenting dendritic cell line (DC2.4) in comparison with murine embryonic fibroblasts (MEFs), both at steady state and after IFN $\alpha$ treatment. Known lipidated and IFN-induced proteins, IFITM3, bone marrow stromal antigen 2 (BST2) and immunity related GTPase M1 (IRGM1), were detected in both cell types after IFN $\alpha$ treatment. We also detected novel palmitoylated proteins expressed at steady state, and validated several of these proteins, including T-lymphocyte activation antigen CD86 and Toll-like receptor 2 (TLR2) in dendritic cells (DCs), and E3 ubiquitin-protein ligase NEDD4 in MEFs. Given the complete novelty of the discovery of a lipid modification occurring on a member of the extensively studied TLR family, we chose to focus further on determining the effects of palmitoylation on TLR2. 
TLRs are critical for the cellular recognition of most pathogens [18]. Their detection of microbial products results in activation of the transcription factor NF- $\kappa B$ and production of inflammatory cytokines and other mediators of the immune response [18]. At least 10 human and 11 mouse TLRs have now been identified, which recognize distinct sets of pathogen-associated molecules [19]. TLR2 is expressed primarily on myelomonocytic cells including antigen presenting cells, such as DCs and macrophages [20]. TLR2 detects the widest range of microbial products among the TLRs, including lipomannan from mycobacteria, zymosan from yeast and bacterial lipopeptides typified by the PamCSK synthetic lipopeptides [18,21]. As such, TLR2 knockout mice and humans with deleterious TLR2 polymorphisms are more susceptible to multiple pathogens [22-26]. Thus, TLR2 is a critical component of the innate immune system, and a better understanding of its posttranslational regulation may prove useful in our defense against pathogenic organisms.

\section{Results and discussion \\ Visualization of palmitoylated proteins in MEFs and DC2.4 cells}

Having made the previous discovery of the critical role of palmitoylation of IFITMs in the innate antiviral immune response $[7,14,15]$, we sought to determine whether any additional IFN-induced proteins are regulated by palmitoylation. For our experiments, we chose to use murine antigen presenting cells (DC2.4) and MEFs because these cell lines are responsive to type I IFNs [14], are amenable to labeling with the alk-16 reporter of protein palmitoylation $[6,14]$ and serve as a control for one another in that type I IFN should induce a similar set of proteins in both cell types. Additionally, this analysis would provide a valuable comparison of the general palmitoylomes of two cell types (myeloid and non-myeloid) with unique functions. DC2.4 cells and MEFs were either left untreated or were treated with IFN $\alpha$ for four hours prior to metabolic labeling with alk-16 in the presence or absence of IFN $\alpha$ for an additional two hours. Cell lysates were reacted with azrho or az-biotin via click chemistry (Figure 1A). Az-rholabeled proteins were separated by SDS-PAGE and visualized by fluorescence gel scanning (Figure 1B). Anti-IFITM3 and anti-GAPDH blotting were used as controls for the activity of IFN $\alpha$ on these cells and for loading, respectively. The fluorescent profiles of the two cell types did not indicate that drastic changes to palmitoylation occur upon IFNa treatment. However, one specific band could be seen uniquely in the IFN $\alpha$ treated samples, appearing at approximately $15 \mathrm{kDa}$, potentially corresponding to the molecular weight of the IFITMs (Figure 1B).

\section{Mass spectrometric identification of palmitoylated proteins in MEFs and DC2.4 cells}

Given that differences induced by IFN $\alpha$ could possibly be masked by highly abundant proteins within the fluorescent palmitoylome profiles observed in Figure 1B, we next utilized the fraction of our samples that had been reacted with az-biotin in order to affinity purify alk-16labeled palmitoylated proteins using streptavidin-coated agarose. These purified proteins were separated by SDSPAGE (Figure 1C) and subjected to in-gel trypsin digestion. Extracted peptides were then identified by tandem mass spectrometry (MS/MS) analysis and assigned to proteins using Mascot software. After subtraction of proteins identified in the control lanes as described in the Methods Section and eliminating proteins with fewer than three spectral counts, a total of 247 proteins were identified from the DC2.4 samples and 437 proteins were identified from the MEF samples, indicating that there is a large difference in the repertoire of palmitoylated proteins expressed by these different cell types (see Additional file 1: Table S1, S2, Figure 1D). Of the proteins identified in DC2.4 cells, 76\% had been previously identified as palmitoylated proteins in our past analysis of this cell line, and by others in proteomic studies of other cell types [7,16,17,27-31] (see Additional file 1: Table S1). Of the proteins identified in MEFs, which had not been palmitoylome profiled before, 64\% were previously reported to be palmitoylated, providing strong validation of our methodology (see Additional file 1: Table S2).

Analysis of several known palmitoylated proteins that were identified uniquely in only one of the two cell types would suggest that their differential detection is due to cell type-specific expression differences. For example, sortilin1 (SORT1), inducible nitric oxide synthase (NOS2) and HLA class II histocompatibility antigen gamma chain (CD74) were uniquely detected in DC2.4 cells, consistent with published data curated by BioGPS showing that murine DCs express these genes while MEFs do not [32-34] (Figure 1D). Conversely, catenin delta (CTNND1), flotillin-2 (FLOT2), GTPase HRAS and tyrosine-protein kinase YES were detected in MEFs, but not in DCs (Figure 1D), again corresponding to cell type-specific expression patterns [32-34]. Further supporting the assertion that the observed differences in the proteins detected are not due to differences in the ability of the two cell types to palmitoylate different subsets of proteins, BioGPS was also utilized to examine the MEF and DC expression patterns for the DHHC palmitoyltransferases (hereafter referred to as DHHCs), the enzyme family responsible for the majority of S-palmitoylation in cells [35,36]. Indeed, murine MEFs and DCs each express nearly all of the DHHCs, with the exception of DHHCs 11 and 19 which are testisspecific and DHHC22 which is brain-specific [32-34]. 


\section{Bioinformatic classification of alk-16-labeled proteins commonly identified in both MEFs and DC2.4 cells}

We identified 121 alk-16-labeled proteins that were common to both DC2.4 cells and MEFs (listed in Additional file 1: Table S3), suggesting that these proteins may perform fundamental cellular functions. These included critical cellular proteins such as calnexin (CANX), GTPase NRAS and the cation-dependent mannose-6-phosphate receptor (M6PR) (Figure 1D). Indeed, exploring the classification of these 121 proteins shows that proteins with enzymatic activity constituted $40 \%$ of the 121 . Transporters and ion channels formed another 20\%. An additional 37\% constituted structural proteins and those in ubiquination and glycosolation processes, with the remaining 3\% being cellular receptors and transcription regulators (see Additional file 2: Figure S2A). Enrichment analysis using Ingenuity defined pathways recapitulated that palmitoylation is critical for a multitude of signaling pathways, including those downstream of ephrin receptors and integrins, as well as axonal guidance signaling (see Additional file 1: Table S4). Similarly, pathways centering on the cellular morphology-controlling small GTPases, RAC1 and/or RHOA, involving multiple palmitoylated proteins were identified (see Additional file 1: Table S4).

On a more global level, network analysis of the 121 shared palmitoylated proteins demonstrated that the MYC proto-oncogene protein and the cellular tumor antigen p53 are upstream of 35 (29\%) of these proteins, which is more than would be expected by chance $(P<0.001$, Additional file 2: Figure S2B). Several DHHCs have been discovered to be misregulated in various tumors, but their contribution to tumorigenesis is currently unclear [37]. Palmitoylated MYC and p53 effector proteins may offer candidates for future experiments determining the role of specific DHHCs and palmitoylated proteins in cancer progression. Taken together, a multitude of coherent pathways are regulated by palmitoylation in both DCs and MEFs, and these data add to our growing appreciation of the roles palmitoylation plays in regulating a myriad of basic cellular processes.

\section{IFN-induced fatty acylated proteins}

We also identified proteins present only in the IFN $\alpha$ treated samples or that had peptide peak area measurements that were increased in the IFN $\alpha$-treated samples by at least 2.5 fold. Since type I IFNs generally induce a common set of antiviral proteins even in distinct cell types, we reasoned that true IFN-induced palmitoylated proteins should be present in the MS/MS results of both DC2.4 cells and MEFs. With this additional criterion, we were left with only three proteins enriched in the IFN $\alpha$-treated samples in both cell types: IFITM3, IRGM1 and BST2, also known as tetherin. Indeed, each of these proteins is known to be IFN-inducible, and IFITM3 serves as a control since we expected to identify this protein based on our past work [7]. Palmitoylation of IRGM1 was verified in our laboratory (see Additional file 2: Figure S1), and was also recently reported to be important for its membrane localization and ability to affect mitochondria membrane dynamics [38]. Interestingly, past palmitoylome profiling studies of murine T cells [17] and human B cells [16] also identified BST2 as a putative palmitoylated protein. Although BST2 palmitoylation has not yet been validated by visualization methods, the study performed in murine $\mathrm{T}$ cells found that mass spectrometric detection of alk-16-labeled BST2 was diminished upon sample treatment with hydroxylamine, which cleaves thioester bonds characteristic of palmitoylation [17]. Likewise, the work identifying BST2 in B cells utilized acyl-biotin exchange chemistry, which allows specific enrichment of S-acylated proteins [16]. BST2 is also well characterized to be lipidated with a GPI anchor [39], which could incorporate long chain fatty acids such as alk-16, although previous alk-16 proteomic profiling studies have not been significantly contaminated with GPI anchored proteins, arguing against this possibility $[7,9,11,17,27]$. Mutation of individual residues within BST2 will be necessary to determine the precise location and type of lipidation reported by alk16 labeling. Nonetheless, our data confirm that the IFNinduced effector proteins, IFITM3, IRGM1 and BST2, are each lipid-modified.

\section{Novel palmitoylated proteins identified in MEFs and DC2.4 cells}

Our work identified 168 proteins in MEFs and 59 proteins in DC2.4 cells that had not been previously reported to be palmitoylated in past proteomic studies (see Additional file 1: Table S1, S2). Many of these proteins were found in only one of the two cell types. For example, we observed the E3 ubiquitin-protein ligase NEDD4 specifically in MEFs (Figure 1D), and confirmed its palmitoylation, thereby identifying an entirely new modification with potential regulatory activity on this extensively studied and critically important protein [40,41] (see Additional file 2: Figure S3). Of particular interest to our laboratory were two proteins uniquely identified in DC2.4 cells that are essential to immune responses, CD86 and TLR2. CD86 is expressed on the surface of mature DCs and provides a costimulatory signal to $\mathrm{T}$ cells that is necessary for their proper activation [42]. We thus sought to confirm CD86 palmitoylation and map its site of modification. Utilizing a C-terminally HA-tagged construct of murine CD86 (CD86-HA), we were able to validate our MS/MS results by demonstrating robust labeling of CD86-HA with alk-16 (Figure 2A). Interestingly, palmitoylated CD86-HA was observed as multiple high MW bands indicating that its glycosylated 


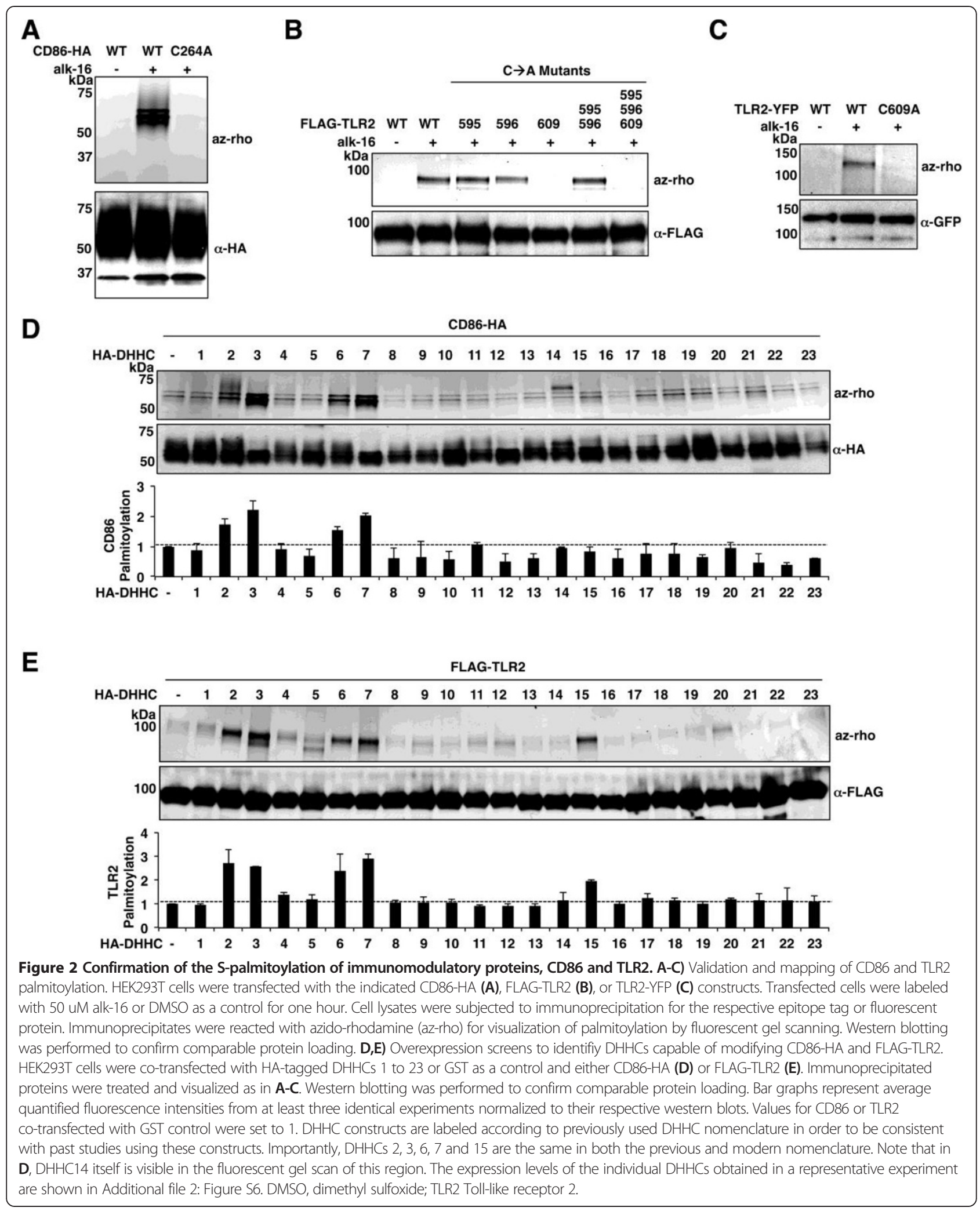

forms are palmitoylated [43], while its non-glycosylated form at the expected molecular weight of $35 \mathrm{kDa}$ was not modified, potentially suggesting sequential modification
(Figure 2A). Mutation of Cys-264 to Ala at the cytoplasmic edge of the CD86 transmembrane domain resulted in a complete loss of alk-16 labeling, implicating Cys-264 as 
the primary site of CD86 palmitoylation (Figure 2A). CD86 has homology to, and a partially redundant function with, T-lymphocyte activation antigen CD80 [44]. We thus generated a CD80-HA construct and also confirmed its palmitoylation (see Additional file 2: Figure S4), thereby demonstrating a conservation of this PTM on the CD80 and CD86 costimulatory molecules.

We similarly confirmed TLR2 palmitoylation utilizing a previously generated construct in which the human TLR2 ER signal sequence was replaced by an artificial signal sequence followed by a FLAG epitope tag $[45,46]$. Indeed, FLAG-TLR2 was readily labeled by alk-16 (Figure 2B). Two cytoplasmic-facing cysteines within TLR2, Cys-595 and Cys-596, were predicted by the CSS-Palm version 3.0 palmitoylation site prediction program [47] to be highly probable sites of palmitoylation. Generating Cys to Ala mutations at these positions and at the nearby Cys-609 allowed for the demonstration that palmitoylation is lost only when Cys-609 is mutated (Figure 2B). Thus, while bioinformatic methods may provide candidate sites of modification, the study of protein palmitoylation requires empirical validation. We further confirmed that Cys-609 is the primary site of human TLR2 S-palmitoylation using a TLR2 construct with its natural ER signal sequence and a C-terminal YFP tag [48,49] (Figure 2C), and also verified that murine TLR2 is S-palmitoylated on the same residue (see Additional file 2: Figure S5).

To determine the extent to which palmitoylation is conserved on TLRs other than TLR2, we tested a panel of human TLRs 1 to 10 for alk-16 labeling in HEK293T cells. While the expression levels of the different TLRs varied, TLR2, TLR5 and TLR10 clearly showed palmitoylation signals above background bands (see Additional file 2: Figure S5). Interestingly, TLR10, which physically interacts with TLR2 and has been associated with regulating TLR2 responses $(43,44)$, had the strongest palmitoylation signal relative to its total protein level (see Additional file 2: Figure S5). These findings reveal that palmitoylation is at least partially conserved on a subset of the human TLRs. These data also confirm that palmitoylation of transmembrane proteins occurs with specificity that is not currently predictable by sequence analysis or by homology with known palmitoylated proteins.

\section{Palmitoylation of CD86 and TLR2 can be increased by specific DHHCs}

In order to examine whether or not S-palmitoylation of CD86 and TLR2 could be installed by specific DHHCs, we performed overexpression screens of each of the DHHCs with CD86 and TLR2. This method is commonly used to identify DHHCs capable of modifying individual protein substrates [35,50-53]. CD86-HA S-palmitoylation was increased by DHHCs 2, 3, 6 and 7 (Figure 2D, Additional file 2: Figure S7). FLAG-TLR2 S-palmitoylation was similarly enhanced by the DHHCs 2, 3, 6 and 7 (Figure 2E, Additional file 2: Figure S7). Since the palmitoylation sites of CD86 and TLR2 are both at the cytoplasmic edge of their respective transmembrane domains, this may suggest that this subset of DHHCs is particularly adept at modifying cysteines adjacent to membranes. However, unlike CD86, palmitoylation of TLR2 was also increased by DHHC15, demonstrating substrate specificity for individual DHHCs (Figure 2E). Overall, these experiments further validate that CD86 and TLR2 are S-palmitoylated proteins whose modification can be mediated enzymatically by specific DHHCs.

\section{Palmitoylation is required for complete activation of NF-KB by TLR2}

Given our laboratory's focus on innate immunity, we chose to further investigate the effects of S-palmitoylation on the activity of TLR2 in primary cultured DCs. For these experiments, we utilized a commonly used covalent inhibitor of DHHCs, 2-bromopalmitate (2-BP) [54]. We found that 2$\mathrm{BP}$ treatment of murine bone marrow-derived (BM) DCs resulted in a loss of the endogenous TLR2 S-palmitoylation signal (Figure 3A). Concomitantly, 2-BP-treated BMDCs lost the ability to produce high levels of the pro-inflammatory cytokines IL-6 and TNF $\alpha$ in response to $\mathrm{Pam}_{3} \mathrm{CSK}_{4}$ and lipomannan, which are microbe-based ligands specifically detected by TLR2 (Figure 3B). Since prolonged exposure to 2-BP can result in off-target effects and cellular toxicity $[55,56]$, we also employed Sendai virus, a TLR-independent activator of cytoplasmic RIG-I-like receptors, as a control [57-61]. Sendai virus induced high levels of IL-6 and TNF $\alpha$ secretion even in the presence of 2-BP (although TNF $\alpha$ was partially affected), indicating that the effects of 2-BP were largely specific to the TLR2 pathway (Figure 3B). Additionally, we did not observe overt cellular toxicity upon 2-BP treatment in the time period examined, as judged by Trypan blue exclusion. Treatment with 2-BP also inhibited lipomannan-induced IL-6 and TNF $\alpha$ secretion from primary human monocyte-derived DCs (see Additional file 2: Figure S8). Thus, the inhibition of cellular palmitoylation by 2-BP leads to an impaired TLR2-dependent proinflammatory cytokine response by DCs.

We next measured the ability of TLR2-YFP and TLR2C609A-YFP to stimulate NF- $\mathrm{kB}$-dependent gene expression in response to microbial ligands. TLR2-C609A-YFP was significantly less able than TLR2-YFP to induce NF-kBdependent luciferase production in response to $\mathrm{Pam}_{3} \mathrm{CSK}_{4}$, lipomannan and zymosan (Figure 3C). Levels of WT and mutant TLR2 were comparable in these experiments as measured by Western blotting (Figure 3D). Similar results demonstrating a defect in the induction of NF-kBdependent luciferase activity by the palmitoylation-deficient TLR2-C609A mutant were also obtained when utilizing FLAG-tagged constructs, and further demonstrated that this 


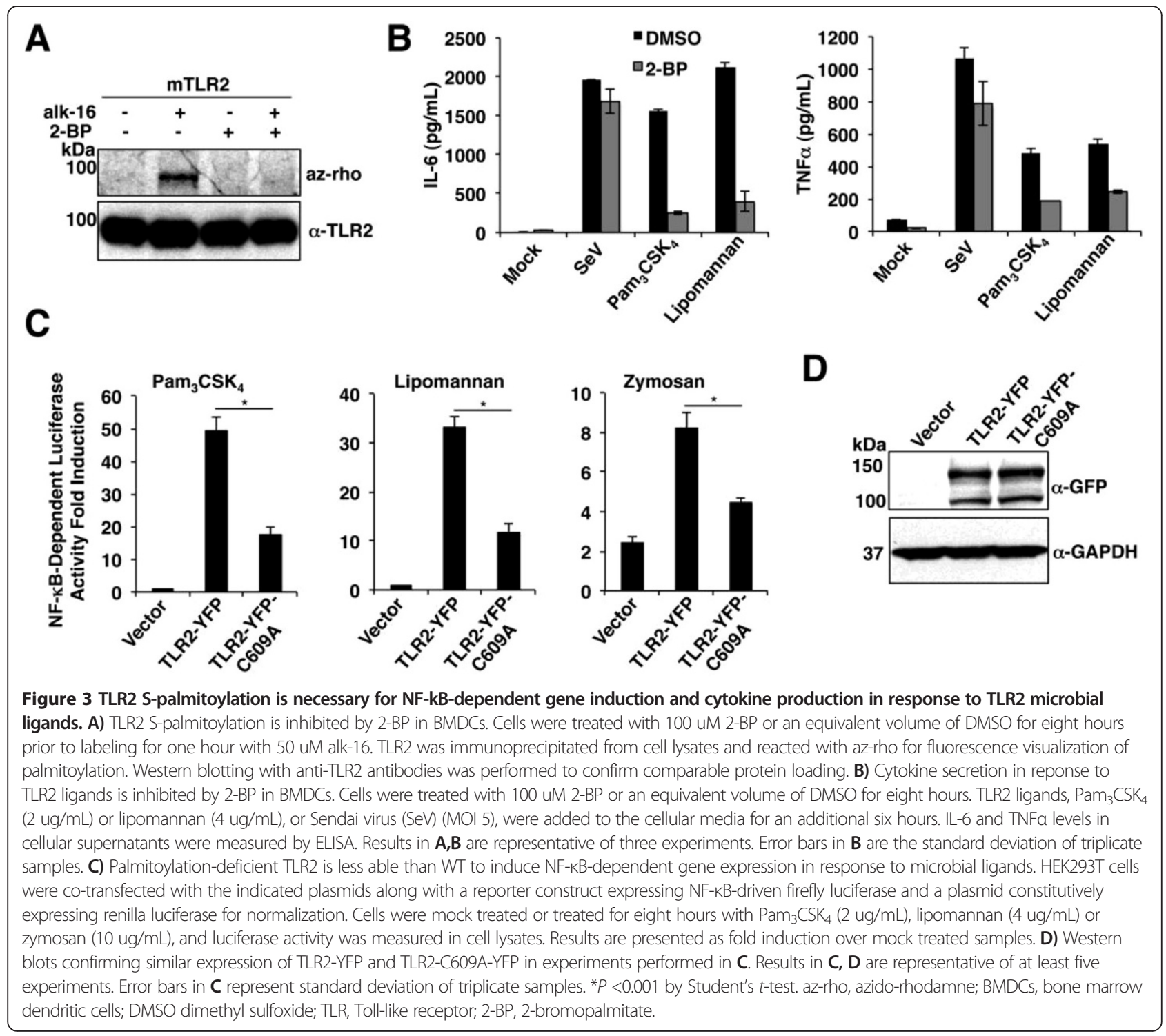

decrease in activity is specific for mutation of the Cys-609 palmitoylation site since a Cys-596 to Ala mutant possessed activity similar to WT FLAG-TLR2 (see Additional file 2: Figure S9). In sum, our data demonstrate that the TLR2 Cys-609 S-palmitoylation site is required for proper activation of NF- $\mathrm{kB}$ in response to microbial ligands.

\section{S-pamitoylation of TLR2 promotes its cell surface localization}

We next examined the effect of endogenous TLR2 ligation on its S-palmitoylation status. We observed that TLR2 palmitoylation is decreased upon stimulation of murine BMDCs with lipomannan (Figure 4A,B). This palmitoylation decrease correlates with a decrease in TLR2 surface staining, while surface staining of costimulatory molecules and MHC II increased as expected for activated DCs (Figure 4C). This finding is also consistent with past observations of TLR2 downregulation at the cell surface upon binding of its ligands [62-64]. The correlation between decreased TLR2 Spalmitoylation and decreased cell surface levels suggested that, perhaps, S-palmitoylation affects TLR2 localization. Indeed, inhibition of palmitoylation with 2-BP decreased endogenous TLR2 surface staining of BMDCs, while overall cellular levels of TLR2 remained similar (Figure 4D). In accord with these results, overexpressed murine FLAGTLR2-C609A showed a subtle, but reproducible, decrease in cell surface staining when compared to WT FLAG-TLR2 (Figure 4E), with their mean fluorescence intensities differing by approximately $25 \%$ on average (Figure $4 \mathrm{~F}$ ). Importantly, achieving robust cell surface staining of FLAG-TLR2 constructs required transfection of five to ten times more DNA than was required for activity assays, perhaps suggesting that the forced overexpression of the WT and C609A TLR2 constructs results in underestimation of their cell 


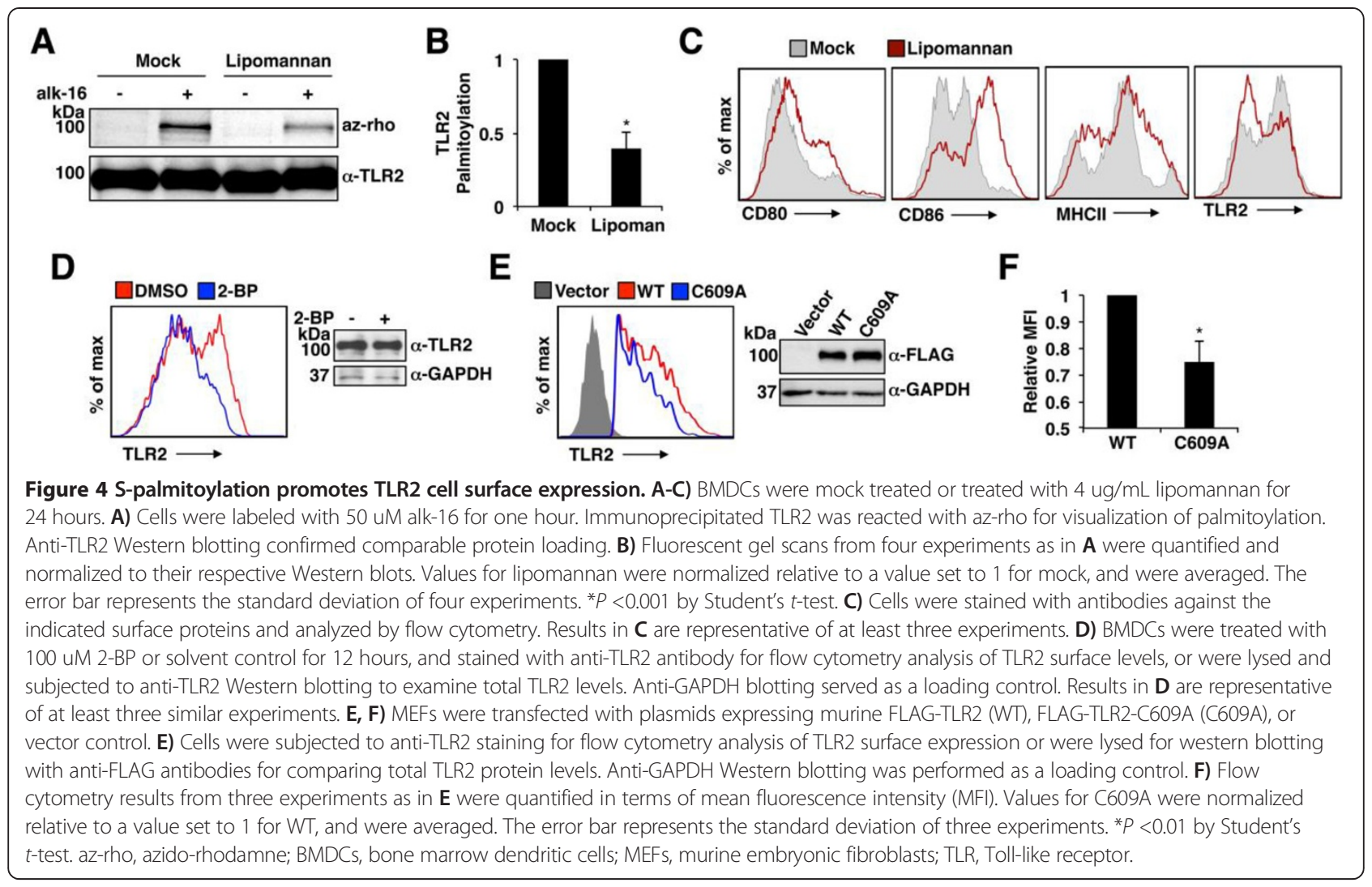

surface differences. We further confirmed that overall protein levels and the stability of TLR2 are not affected by palmitoylation by performing pulse-chase analysis of FLAGTLR2 and FLAG-TLR2-C609A, which demonstrated that the decay of the WT and mutant proteins was similar (see Additional file 2: Figure S10). Overall, we conclude that S-palmitoylation positively regulates TLR2 activity, at least in part, by promoting its trafficking to the cell surface.

\section{Conclusions}

The development of chemical proteomic methods to identify palmitoylated proteins has led to a tremendous increase in our appreciation of the numerous ways in which palmitoylation regulates key cellular processes and can control cell type-specific pathways $[7,9,11,16,17,28-31,65]$. We previously reported the identification of 157 palmitoylated proteins in DC2.4 cells that included proteins critical to antiviral immune responses [7]. However, the absence of several expected palmitoylated proteins from our MS/ MS identification led us to hypothesize that additional unknown palmitoylated proteins also remained to be identified in this cell type [13]. Indeed, refinements to our palmitoylated protein enrichment protocol that included increased incubation time with streptavidin agarose and repeated washing steps, along with updates to our mass spectrometry capabilities, have led to a deeper coverage of the DC2.4 palmitoylome (Figure 1 and Additional file 1:
Table S1). In comparison to the palmitoylome of MEFs, we identified novel putative palmitoylated proteins that play a role in the immune functions of DCs. Following up on several of these proteins, we were able to validate palmitoylation of the CD80 and CD86 costimulatory molecules (Figure 2A and Additional file 2: Figure S4) as well as TLR2 (Figure 2B,C and Additional file 2: Figure S5). Interestingly, our proteomic results indicated that MEFs express a wider diversity of palmitoylated proteins than DCs (Figure 1D and Additional file 1: Table S1, S2), and include many proteins, such as NEDD4, that should lead to exciting follow up studies.

Our discovery of CD80, CD86 and TLR2 palmitoylation in DCs reveals novel mechanisms of immune regulation, and underscores the value of examining palmitoylation in diverse cells types. CD80 and CD86 are essential for immune responses to most pathogens as well as for elimination of tumor cells by the immune system [66]. These costimulatory molecules provide a second signal for T-cell activation in addition to $\mathrm{MHC} /$ peptide complexes presented on the surface of mature DCs [66]. Future studies will aim to determine whether palmitoylation is involved in the trafficking of these molecules to the DC surface, their subsequent clustering at the immunological synapse and their impact on immune function.

Here, we specifically followed up on the role of S-palmitoylation in regulating TLR2 activity. Indeed, the 
response to all microbial ligands of TLR2 that were tested was decreased in the absence of TLR2 S-palmitoylation, indicating that this lipid modification is a positive regulator of TLR2 activity (Figure 3 and Additional file 2: Figure S8, S9). S-palmitoylation is necessary for complete localization of TLR2 at the cell surface, and thus likely affects its interactions with its ligands (Figure 4D-F). While an effect of palmitoylation on the cell surface expression of multiple other proteins has been previously reported [3], it is interesting to note that although CD86 and TLR2 are both S-palmitoylated at membrane-proximal cysteines (Figure 2A-C) by a similar subset of DHHCs (Figure 2D,E), their cell surface localization is inversely regulated during DC activation (Figure 4B). This reinforces the commonly observed theme that palmitoylation can have unpredictable effects depending on the protein context in which it occurs $[1,3,67]$. Future analyses of how S-palmitoylation of additional immune receptors controls their localization, interactions and signaling will be an exciting area of future study, as will the examination of potential subversion of TLR2 S-palmitoylation by pathogens. Importantly, the identification of S-palmitoylation of TLRs suggests that this reversible form of protein lipidation [68] may be targeted in the future to manipulate beneficial and non-beneficial TLR2-mediated immune responses.

\section{Methods}

Click chemistry, fluorescence gel scanning, and proteomic identification of palmitoylated proteins

Click chemistry was performed as previously described [6-8,69]. In short, immunoprecipitated proteins or cell lysates were reacted for one hour at room temperature with $100 \mu \mathrm{M}$ azido-rhodamine [6] or azido-azo-biotin [12], $1 \mathrm{mM}$ tris(2-carboxyethyl)phosphine hydrochloride (TCEP), $100 \mu \mathrm{M} \quad$ tris[(1-benzyl-1H-1,2,3-triazol-4-yl)methyl]amine (TBTA), and $1 \mathrm{mM} \mathrm{CuSO}_{4} \cdot 5 \mathrm{H}_{2} \mathrm{O}$. In-gel fluorescence scanning was performed using a Typhoon 9400 imager (Amersham Biosciences, Amersham, United Kingdom) (excitation $532 \mathrm{~nm}, 580 \mathrm{~nm}$ detection filter). Enrichment of alk-16labeled proteins was performed according to a standard protocol [7-9] with minor adjustments in the amount of protein and buffers used and the number of washing steps, in order to increase specificity and recovery of labeled proteins. Proteins in cell lysates reacted with azido-azo-biotin were methanol precipitated at $-20^{\circ} \mathrm{C}$ overnight, washed with ice-cold methanol three times, dried, and resolubilized in SDS buffer (4\% SDS, $150 \mathrm{mM} \mathrm{NaCl}, 50 \mathrm{mM}$ triethanolamine, $\mathrm{pH}$ 7.4). A total of $2.5 \mathrm{mg}$ of the resolubilized protein in $1 \mathrm{~mL}$ was diluted to $15 \mathrm{~mL}$ with PBS containing $1 \%$ Brij97 and was incubated with $100 \mu \mathrm{L}$ of pre-washed highcapacity streptavidin agarose (Thermo Scientific, Waltham, Massachusetts, United States) at $4^{\circ} \mathrm{C}$ on a nutating mixer overnight. The streptavidin agarose was then washed five times with $15 \mathrm{~mL}$ PBS containing 1\% SDS followed by three washes with $15 \mathrm{~mL}$ PBS and two washes with $250 \mathrm{mM}$ ammonium bicarbonate. Cysteines were then capped with iodoacetamide, followed by three additional washes with $250 \mathrm{mM}$ ammonium bicarbonate. Proteins were eluted from the agarose three times using sodium dithionite, and concentrated using low molecular weight Centricons (Millipore, Billerica, Massachusetts, United States) as previously described [8]. Proteins were then separated by SDSPAGE for in-gel digestion, peptide extraction and MS/MS analysis. MS/MS was performed by the Rockefeller University Proteomics Resource Center on a Thermo LTQOrbitrap mass spectrometer that had undergone recent professional tuning and alignment, and that was equipped with a Dionex 3000 capillary/nano HPLC. MS/MS spectra of peptides were analyzed against the mouse protein database using the Mascot version 2.3 search tool. Data from samples analyzed by Mascot were compared with ProteomeDiscoverer software (Thermo Scientific) utilizing a cutoff score of 40 and a requirement of two peptides per protein. Data were then further manually analyzed by eliminating proteins that were identified with less than a 20 fold detection increase over the controls based on the ProteomeDiscoverer software peptide peak area measurement, and by eliminating proteins with a sum of less than three spectral counts in the alk-16-treated samples. Proteomic lists from other studies were compared with this data set using gene symbols obtained, if necessary, by converting published identifiers into gene symbols using the UniProt database along with the DAVID Gene ID Conversion Tool (National Institute of Allergy and Infectious Diseases, National Insttutes of Health (NIAID, NIH)).

\section{Pathway enrichment and network analysis}

Protein data from DC2.4 and MEF cells with suitable quality as described above were standardized using GenPept accession numbers. Overlapping proteins were subsequently ported into Ingenuity IPA software [70]. Canonical pathway enrichment was performed using IPA defined classes and corrected for multiplicity using the Benjamini-Hochberg method. Network analysis and subsequent diagrams were generated using only direct associations among the proteins. $P$-values for upstream analysis were calculated using the Fisher's exact test.

\section{DNA constructs and luciferase assays}

TLR2-YFP, pCDNA3-YFP and fluorescent protein-tagged constructs for the other human TLRs were obtained from Addgene (plasmids 13014, 13033, 13016, 13641, 13018, 13019, 13020, 13022, 13024, 13642, and 13643, deposited by Doug Golenbock). The human FLAG-TLR2 construct was also obtained from Addgene (plasmid 13082, deposited by Ruslan Medzhitov), as was the mouse FLAG-TLR2 construct (plasmid 12291 deposited by Bruce Beutler [71]). CD80 and CD86 coding sequences were amplified by PCR 
from DC2.4 cell cDNA incorporating a C-terminal HA tag, as well as EcoRI and BglII restriction sites for insertion into the pCAGGS expression vector. IRGM1 coding sequence was amplified by PCR from DC2.4 cell cDNA incorporating SalI and XhoI restriction sites for in-frame insertion into the pCMV-HA expression vector. The QuikChange MultiSite Directed Mutagenesis Kit from Stratagene (La Jolla, California, United States) was used for cysteine mutagenesis. HA-tagged DHHCs and GST control plasmids were kindly provided by Masaki Fukata (NIPS, Okazaki, Japan). For luciferase assays, a NF-kB-dependent firefly luciferase reporter construct provided by Dimitris Thanos (Biomedical Sciences Research Center, Athens, Greece) was used along with the pRL-TK plasmid (Promega, Madison, Wisconsin, United States) driving constitutive expression of Renilla luciferase. The Dual Luciferase Reporter Assay System from Promega was used for detection of firefly and Renilla luciferase. Mycobacterium smegmatis lipomannan, zymosan and $\mathrm{Pam}_{3} \mathrm{CSK}_{4}$ were purchased from InvivoGen (San Diego, California, United States). Sendai virus strain Cantell was grown in 10-day embryonated chicken eggs and titered as previously described [72].

\section{Cell culture and flow cytometry}

DC2.4, MEFs, and HEK293T cells were grown in DMEM supplemented with $10 \% \mathrm{FBS}$ in a $37^{\circ} \mathrm{C}$ humidified incubator with an atmosphere of $5 \% \mathrm{CO}_{2}$. BMDCs were generated by culturing bone marrow cells for 7 days in RPMI supplemented with $10 \% \mathrm{FBS}, 10 \mathrm{ug} / \mathrm{mL}$ gentamicin and $25 \mathrm{ng} /$ $\mathrm{mL}$ GMCSF, with media changes on days 3 and 5 . Nonadherent cells were used for experimentation and were validated to be DCs based on $70 \%$ to $80 \%$ of the cells staining positive for CD11c and MHCII as measured by flow cytometry. Murine IFN $\alpha$ was purchased from eBioscience (San Diego, California, United States). All flow cytometry staining was performed after a 10-minute incubation in ice cold PBS with Fc receptor block (BD Biosciences, San Jose, California, United States). Antibodies from BD Biosciences directed at the following molecules were used at 1:200 dilution in ice cold PBS for 20 minutes at $4^{\circ} \mathrm{C}$ : CD11c (\#557400), MHCII (\#553552), CD80 (\#553769), CD86 (\#553691) and TLR2 (\#562625). All samples were washed three times with ice cold PBS, read on a BD FACSCanto II flow cytometer and analyzed using Flowjo software. For flow cytometry of transfected MEFs, cells were detached from plates by incubating in PBS/10 $\mathrm{mM}$ ethylenediaminetetraacetic acid (EDTA) for 20 minutes at $37^{\circ} \mathrm{C}$.

Transfections, metabolic labeling, cell lysis, immunoprecipitations, and Western blotting

HEK293T cells were transfected overnight at 60\% confluency using Lipofectamine 2000 (Life Technologies, Carlsbad, California, United States) according to the manufacturer's instructions. For TLR2 activity experiments,
$100 \mathrm{ng}$ of TLR2-expressing plasmids were transfected into wells of a 12-well plate along with 1 ug of NF-kB luciferase reporter plasmid and $50 \mathrm{ng}$ of pRL-TK. For all other experiments, $1 \mathrm{ug}$ or $2 \mathrm{ug}$ of plasmid was transfected for 12-well and 6-well plates, respectively. For cell labeling, alk-16 was added to $37^{\circ} \mathrm{C}$ DMEM containing $2 \%$ charcoal/ dextran-filtered fetal bovine serum for a final concentration of $50 \mathrm{uM}$. For mock-labeled samples, an equivalent volume of DMSO rather than alk-16 solution was added to the labeling media. After replacing the media, labeling was allowed to proceed for one to two hours under standard culture conditions at $37^{\circ} \mathrm{C}$ or for four hours for proteomic experiments. 2-BP was purchased from SigmaAldrich (St. Louis, Missouri, United States), prepared fresh in DMSO for each experiment and used at a concentration of $100 \mu \mathrm{M}$. For all alk-16 labeling experiments, cells were washed twice with room temperature PBS and pellets were flash-frozen with liquid nitrogen for storage at $-80^{\circ} \mathrm{C}$. Cell pellets were lysed with Brij97 buffer (1\% Brij 97, $150 \mathrm{mM} \mathrm{NaCl}, 50 \mathrm{mM}$ triethanolamine, $\mathrm{pH}$ 7.4) and cellular debris was cleared by centrifugation for five minutes at 1,000 x g. For immunoprecipitations, anti-FLAG or anti-HA conjugated agarose (Sigma), or anti-GFP antibody (JL-8, Clontech, Mountain View, California, United States) or anti-TLR2 antibody (MAb-mTLR2, InvivoGen, San Diego, California, United States) with protein G agarose (Roche Diagnostics, Indianapolis, Indiana, United States) was used. Western blots for FLAG-tagged proteins were performed with anti-FLAG antibody produced in rabbit (Sigma-Aldrich, St. Louis, Missouri, United States) at a 1:1,000 dilution. Blots for HA-tagged proteins were performed using a polyclonal antibody preparation from Clontech at a 1:1,000 dilution. Blots for fluorescent proteintagged TLRs were performed with Clontech mouse monoclonal anti-GFP (JL-8) at a 1:2,500 dilution and blots for endogenous mouse TLR2 was performed with anti-TLR2 (MAB1530) from R\&D Systems (Minneapolis, Minnesota, United States) at a 1:1,000 dilution.

\section{Availability of supporting data}

MS/MS raw data files have been uploaded to the Peptide Atlas data repository and are freely available for download and analysis. Files for DC2.4 samples can be accessed at PeptideAtlas under dataset identifier PASS00594 [73]. These files correspond to gel slices cut from the first four lanes of Figure 1C. Specifically, files 1 to 7 are from lane 1 (DMSO treated cells), files 8 to 14 are from lane 2 (IFN alpha/DMSO treated cells), files 15 to 21 are from lane 3 (alk-16 treated cells), and files 22 to 28 are from lane 4 (IFN alpha/alk-16 treated cells). Files for MEF samples can be accessed at PeptideAtlas under dataset identifier PASS00595 [74]. These files correspond to gel slices cut from lanes 5 through 8 of 
Figure 1C. Specifically, files 29 to 35 are from lane 5 (DMSO treated cells), files 36 to 42 are from lane 6 (IFN alpha/DMSO treated cells), files 43 to 49 are from lane 7 (alk-16 treated cells), and files 50 to 56 are from lane 8 (IFN alpha/alk-16 treated cells).

\section{Additional files}

Additional file 1: Table S1: Alk-16-labeled proteins identified from DC2.4 cells. Table S2: Alk-16-labeled proteins identified from MEFs. Table S3: Palmitoylated proteins identified in both DC2.4 cells and MEFs. Table S4: Molecular pathway enrichment of palmitoylated proteins found in both MEFs and DC2.4 cells.

Additional file 2: Figure S1: Confirmation of IRGM1 palmitoylation. Figure S2: Ingenuity pathway analysis of palmitoylated proteins identified in both DC2.4 cells and MEFs. Figure S3: Confirmation of NEDD4 palmitoylation. Figure S4: Identification of CD80 palmitoylation. Figure S5: Murine (m) TLR2 is S-palmitoylated on Cys-609. Figure S6: Partial conservation of palmitoylation occurring on the human TLRs. Figure S7: Expression levels of HA-tagged DHHC constructs. Figure S8: TLR2-dependent cytokine responses are decreased by chemical inhibition of palmitoylation in primary human DCs. Figure S9: TLR2 S-palmitoylation is required for NF-kB-dependent gene induction.Figure S10: S-palmitoylation of TLR2 does not affect its stability.

\section{Competing interests}

The authors declare that they have no competing interests.

\section{Authors' contributions}

The study was conceived by $\mathrm{HCH}$ and JSY. HCH supervised proteomic studies and initial validation of TLR2 palmitoylation performed by JSY at the Rockefeller University. Further studies were supervised by JSY at The Ohio State University. JCH performed DHHC overexpression experiments. JLC performed bioinformatics pathway analysis of palmitoylated proteins. MVSR and LSS generated and performed experiments with human monocyte derived dendritic cells. MRP and BWZ confirmed palmitoylation of NEDD4. JT provided reagents and assistance in the generation and study of murine bone marrow derived dendritic cells. All remaining experiments unless otherwise mentioned above were performed by NMC. The manuscript was drafted by JSY with significant editorial contributions from NMC, $\mathrm{HCH}$, and LSS. All authors read and approved the final manuscript.

\section{Acknowledgements}

This work was supported by funding from the NIH/NIAID (grant R00AI095348 to J.S.Y.), the NIH/NIGMS (R01GM087544 to HCH), and the Ohio State University Public Health Preparedness for Infectious Diseases (PHPID) program. NMC is supported by the Ohio State University Systems and Integrative Biology Training Program (NIH/NIGMS grant T32GM068412). BWZ is a fellow of the National Science Foundation Graduate Research Fellowship Program (DGE-0937362). The authors thank Dr. Masaki Fukata (NIPS, Japan) for providing DHHC constructs. Partial funding for Open Access provided by The Ohio State University Open Access Fund.

\section{Author details}

${ }^{1}$ Department of Microbial Infection and Immunity, Center for Microbial Interface Biology, The Ohio State University, Columbus, OH 43210, USA. ${ }^{2}$ Biomedical Informatics, Internal Medicine in the Division of Medical Oncology, The Ohio State University, Columbus, OH 43210, USA.

${ }^{3}$ Departments of Chemistry and Molecular and Computational Biology, University of Southern California, Los Angeles, CA 90089, USA. " Laboratory of Chemical Biology and Microbial Pathogenesis, Rockefeller University, New York, NY 10065, USA.

Received: 11 August 2014 Accepted: 20 October 2014

Published online: 05 November 2014

\section{References}

1. Salaun C, Greaves J, Chamberlain LH: The intracellular dynamic of protein palmitoylation. J Cell Biol 2010, 191:1229-1238.

2. Charollais J, Van Der Goot FG: Palmitoylation of membrane proteins (Review). Mol Membr Biol 2009, 26:55-66.

3. Blaskovic S, Blanc M, van der Goot FG: What does S-palmitoylation do to membrane proteins? FEBS J 2013, 280:2766-2774.

4. Hang HC, Linder ME: Exploring protein lipidation with chemical biology. Chem Rev 2011, 111:6341-6358.

5. Tom CT, Martin BR: Fat chance! Getting a grip on a slippery modification. ACS Chem Biol 2013, 8:46-57.

6. Charron G, Zhang MM, Yount JS, Wilson J, Raghavan AS, Shamir E, Hang HC: Robust fluorescent detection of protein fatty-acylation with chemical reporters. J Am Chem Soc 2009, 131:4967-4975.

7. Yount JS, Moltedo B, Yang YY, Charron G, Moran TM, Lopez CB, Hang HC: Palmitoylome profiling reveals S-palmitoylation-dependent antiviral activity of IFITM3. Nat Chem Biol 2010, 6:610-614.

8. Yount JS, Zhang MM, Hang HC: Visualization and identification of fatty acylated proteins using chemical reporters. Curr Protoc Chem Biol 2011 3:65-79.

9. Yount JS, Charron G, Hang HC: Bioorthogonal proteomics of 15hexadecynyloxyacetic acid chemical reporter reveals preferential targeting of fatty acid modified proteins and biosynthetic enzymes. Bioorg Med Chem 2012, 20:650-654.

10. Hannoush RN, Arenas-Ramirez N: Imaging the lipidome: omega-alkynyl fatty acids for detection and cellular visualization of lipid-modified proteins. ACS Chem Biol 2009, 4:581-587.

11. Martin BR, Cravatt BF: Large-scale profiling of protein palmitoylation in mammalian cells. Nat Methods 2009, 6:135-138.

12. Yang YY, Grammel M, Raghavan AS, Charron G, Hang HC: Comparative analysis of cleavable azobenzene-based affinity tags for bioorthogonal chemical proteomics. Chem Biol 2010, 17:1212-1222.

13. Yount JS, Zhang MM, Hang HC: Emerging roles for protein Spalmitoylation in immunity from chemical proteomics. Curr Opin Chem Biol 2013, 17:27-33.

14. Yount JS, Karssemeijer RA, Hang HC: S-palmitoylation and ubiquitination differentially regulate interferon-induced transmembrane protein 3 (IFITM3)-mediated resistance to influenza virus. J Biol Chem 2012. 287:19631-19641.

15. Hach JC, McMichael T, Chesarino NM, Yount JS: Palmitoylation on conserved and non-conserved cysteines of murine IFITM1 regulates its stability and anti-influenza A virus activity. J Virol 2013, 87:9923-9927.

16. Ivaldi C, Martin BR, Kieffer-Jaquinod S, Chapel A, Levade T, Garin J, Journet A: Proteomic analysis of S-acylated proteins in human B cells reveals palmitoylation of the immune regulators CD20 and CD23. PLoS One 2012, 7:e37187.

17. Martin BR, Wang C, Adibekian A, Tully SE, Cravatt BF: Global profiling of dynamic protein palmitoylation. Nat Methods 2011, 9:84-89.

18. Kawai T, Akira S: The roles of TLRs, RLRs and NLRs in pathogen recognition. Int Immunol 2009, 21:317-337.

19. Kawai T, Akira S: The role of pattern-recognition receptors in innate immunity: update on Toll-like receptors. Nat Immunol 2010, 11:373-384.

20. Muzio M, Bosisio D, Polentarutti N, D'amico G, Stoppacciaro A, Mancinelli R, van't Veer C, Penton-Rol G, Ruco LP, Allavena P, Mantovani A: Differential expression and regulation of toll-like receptors (TLR) in human leukocytes: selective expression of TLR3 in dendritic cells. J Immunol 2000, 164:5998-6004.

21. Borrello S, Nicolo C, Delogu G, Pandolfi F, Ria F: TIr2: a crossroads between infections and autoimmunity? Int I Immunopathol Pharmacol 2011 24:549-556.

22. Takeuchi O, Hoshino K, Akira S: Cutting edge: TLR2-deficient and MyD88deficient mice are highly susceptible to Staphylococcus aureus infection. J Immunol 2000, 165:5392-5396.

23. Hawn TR, Smith KD, Aderem A, Skerrett SJ: Myeloid differentiation primary response gene (88)- and toll-like receptor 2-deficient mice are susceptible to infection with aerosolized Legionella pneumophila. J Infect Dis 2006, 193:1693-1702.

24. Bafica A, Scanga CA, Feng CG, Leifer C, Cheever A, Sher A: TLR9 regulates Th1 responses and cooperates with TLR2 in mediating optimal resistance to Mycobacterium tuberculosis. J Exp Med 2005, 202:1715-1724. 
25. Thuong NT, Hawn TR, Thwaites GE, Chau TT, Lan NT, Quy HT, Hieu NT, Aderem A, Hien TT, Farrar JJ, Dunstan SJ: A polymorphism in human TLR2 is associated with increased susceptibility to tuberculous meningitis. Genes Immun 2007, 8:422-428.

26. Lorenz E, Mira JP, Cornish KL, Arbour NC, Schwartz DA: A novel polymorphism in the toll-like receptor 2 gene and its potential association with staphylococcal infection. Infect Immun 2000, 68:6398-6401.

27. Wilson JP, Raghavan AS, Yang YY, Charron G, Hang HC: Proteomic analysis of fatty-acylated proteins in mammalian cells with chemical reporters reveals S-acylation of histone H3 variants. Mol Cell Proteomics 2011, 10:M110 001198.

28. Dowal L, Yang W, Freeman MR, Steen H, Flaumenhaft R: Proteomic analysis of palmitoylated platelet proteins. Blood 2011, 118:e62-e73.

29. Li Y, Martin BR, Cravatt BF, Hofmann SL: DHHC5 protein palmitoylates flotillin-2 and is rapidly degraded on induction of neuronal differentiation in cultured cells. J Biol Chem 2012, 287:523-530.

30. Merrick BA, Dhungana S, Williams JG, Aloor JJ, Peddada S, Tomer KB, Fessler MB: Proteomic profiling of S-acylated macrophage proteins identifies a role for palmitoylation in mitochondrial targeting of phospholipid scramblase 3. Mol Cell Proteomics 2011, 10:M110 006007.

31. Kang R, Wan J, Arstikaitis P, Takahashi H, Huang K, Bailey AO, Thompson JX, Roth AF, Drisdel RC, Mastro R, Green WN, Yates JR 3rd, Davis NG, El-Husseini A: Neural palmitoyl-proteomics reveals dynamic synaptic palmitoylation. Nature 2008, 456:904-909.

32. Wu C, Orozco C, Boyer J, Leglise M, Goodale J, Batalov S, Hodge CL, Haase J, Janes J, Huss JW 3rd, Su Al: BioGPS: an extensible and customizable portal for querying and organizing gene annotation resources. Genome Biol 2009, 10:R130.

33. Su Al, Wiltshire T, Batalov S, Lapp H, Ching KA, Block D, Zhang J, Soden R, Hayakawa M, Kreiman G, Cooke MP, Walker JR, Hogenesch JB: A gene atlas of the mouse and human protein-encoding transcriptomes. Proc Natl Acad Sci U S A 2004, 101:6062-6067.

34. Su Al, Cooke MP, Ching KA, Hakak Y, Walker JR, Wiltshire T, Orth AP, Vega RG, Sapinoso LM, Moqrich A, Patapoutian A, Hampton GM, Schultz PG, Hogenesch JB: Large-scale analysis of the human and mouse transcriptomes. Proc Natl Acad Sci U S A 2002, 99:4465-4470.

35. Greaves J, Chamberlain LH: DHHC palmitoyl transferases: substrate interactions and (patho)physiology. Trends Biochem Sci 2011, 36:245-253.

36. Linder ME, Jennings BC: Mechanism and function of DHHC Sacyltransferases. Biochem Soc Trans 2013, 41:29-34.

37. Greaves J, Chamberlain LH: New links between S-acylation and cancer. J Pathol 2014, 233:4-6.

38. Henry SC, Schmidt EA, Fessler MB, Taylor GA: Palmitoylation of the immunity related GTPase, Irgm 1: impact on membrane localization and ability to promote mitochondrial fission. PLoS One 2014, 9:e95021.

39. Kupzig S, Korolchuk V, Rollason R, Sugden A, Wilde A, Banting G: Bst-2/ HM1.24 is a raft-associated apical membrane protein with an unusual topology. Traffic 2003, 4:694-709.

40. Rotin D, Kumar S: Physiological functions of the HECT family of ubiquitin ligases. Nat Rev Mol Cell Biol 2009, 10:398-409.

41. Scheffner M, Kumar S: Mammalian HECT ubiquitin-protein ligases: biological and pathophysiological aspects. Biochim Biophys Acta 2014, 1843:61-74

42. Imro MA, Dellabona P, Manici S, Heltai S, Consogno G, Bellone M, Rugarli C, Protti MP: Human melanoma cells transfected with the B7-2 costimulatory molecule induce tumor-specific CD8+ cytotoxic T lymphocytes in vitro. Hum Gene Ther 1998, 9:1335-1344.

43. Hollsberg P, Scholz C, Anderson DE, Greenfield EA, Kuchroo VK, Freeman GJ, Hafler DA: Expression of a hypoglycosylated form of CD86 (B7-2) on human T cells with altered binding properties to CD28 and CTLA-4. J Immunol 1997, 159:4799-4805.

44. Freeman GJ, Gribben JG, Boussiotis VA, Ng JW, Restivo VA Jr, Lombard LA, Gray GS, Nadler LM: Cloning of B7-2: a CTLA-4 counter-receptor that costimulates human T cell proliferation. Science 1993, 262:909-911.

45. Bulut Y, Faure E, Thomas L, Equils O, Arditi M: Cooperation of Toll-like receptor 2 and 6 for cellular activation by soluble tuberculosis factor and Borrelia burgdorferi outer surface protein A lipoprotein: role of Tollinteracting protein and IL-1 receptor signaling molecules in Toll-like receptor 2 signaling. J Immunol 2001, 167:987-994.

46. Yamamoto M, Sato S, Mori K, Hoshino K, Takeuchi O, Takeda K, Akira S: Cutting edge: a novel Toll/IL-1 receptor domain-containing adapter that preferentially activates the IFN-beta promoter in the Toll-like receptor signaling. J Immunol 2002, 169:6668-6672.

47. Ren J, Wen L, Gao X, Jin C, Xue Y, Yao X: CSS-Palm 2.0: an updated software for palmitoylation sites prediction. Protein Eng Des Sel 2008 21:639-644.

48. Latz E, Visintin A, Lien E, Fitzgerald KA, Monks BG, Kurt-Jones EA, Golenbock DT, Espevik T: Lipopolysaccharide rapidly traffics to and from the Golgi apparatus with the toll-like receptor 4-MD-2-CD14 complex in a process that is distinct from the initiation of signal transduction. $J$ Biol Chem 2002, 277:47834-47843.

49. Sandor F, Latz E, Re F, Mandell L, Repik G, Golenbock DT, Espevik T, Kurt-Jones EA, Finberg RW: Importance of extra- and intracellular domains of TLR1 and TLR2 in NFkappa B signaling. J Cell Biol 2003, 162:1099-1110.

50. Fukata Y, Iwanaga T, Fukata M: Systematic screening for palmitoyl transferase activity of the DHHC protein family in mammalian cells. Methods 2006, 40:177-182.

51. Fukata M, Fukata Y, Adesnik H, Nicoll RA, Bredt DS: Identification of PSD-95 palmitoylating enzymes. Neuron 2004, 44:987-996.

52. Mill $P$, Lee AW, Fukata $Y$, Tsutsumi R, Fukata M, Keighren M, Porter RM McKie L, Smyth I, Jackson IJ: Palmitoylation regulates epidermal homeostasis and hair follicle differentiation. PLoS Genet 2009, 5:e1000748.

53. Tsutsumi R, Fukata Y, Noritake J, Iwanaga T, Perez F, Fukata M: Identification of $\mathrm{G}$ protein alpha subunit-palmitoylating enzyme. Mol Cell Biol 2009, 29:435-447.

54. Webb Y, Hermida-Matsumoto L, Resh MD: Inhibition of protein palmitoylation, raft localization, and T cell signaling by 2-bromopalmitate and polyunsaturated fatty acids. J Biol Chem 2000, 275:261-270.

55. Davda D, El Azzouny MA, Tom CT, Hernandez JL, Majmudar JD, Kennedy RT, Martin BR: Profiling targets of the irreversible palmitoylation inhibitor 2bromopalmitate. ACS Chem Biol 2013, 8:1912-1917.

56. Zheng BH, DeRan M, Li XY, Liao XB, Fukata M, Wu X: 2-Bromopalmitate analogues as activity-based probes to explore palmitoyl acyltransferases. J Am Chem Soc 2013, 135:7082-7085.

57. Yount JS, Kraus TA, Horvath CM, Moran TM, Lopez CB: A novel role for viral-defective interfering particles in enhancing dendritic cell maturation. J Immunol 2006, 177:4503-4513.

58. Yount JS, Moran TM, Lopez CB: Cytokine-independent upregulation of MDA5 in viral infection. J Virol 2007, 81:7316-7319.

59. Yount JS, Gitlin L, Moran TM, Lopez CB: MDA5 participates in the detection of paramyxovirus infection and is essential for the early activation of dendritic cells in response to Sendai Virus defective interfering particles. J Immunol 2008, 180:4910-4918.

60. Kato H, Takeuchi O, Sato S, Yoneyama M, Yamamoto M, Matsui K, Uematsu S, Jung A, Kawai T, Ishii KJ, Yamaguchi O, Otsu K, Tsujimura T, Koh CS, Reis e Sousa C, Matsuura Y, Fujita T, Akira S: Differential roles of MDA5 and RIG-I helicases in the recognition of RNA viruses. Nature 2006, 441:101-105.

61. Lopez CB, Yount JS, Moran TM: Toll-like receptor-independent triggering of dendritic cell maturation by viruses. J Virol 2006, 80:3128-3134.

62. Brandt KJ, Fickentscher C, Kruithof EK, de Moerloose P: TLR2 ligands induce NF-kappaB activation from endosomal compartments of human monocytes. PLoS One 2013, 8:e80743.

63. Barbalat R, Lau L, Locksley RM, Barton GM: Toll-like receptor 2 on inflammatory monocytes induces type I interferon in response to viral but not bacterial ligands. Nat Immuno/ 2009, 10:1200-1207.

64. Triantafilou M, Gamper FG, Haston RM, Mouratis MA, Morath S, Hartung T, Triantafilou K: Membrane sorting of toll-like receptor (TLR)-2/6 and TLR2/ 1 heterodimers at the cell surface determines heterotypic associations with CD36 and intracellular targeting. J Biol Chem 2006, 281:31002-31011.

65. Roth AF, Wan J, Bailey AO, Sun B, Kuchar JA, Green WN, Phinney BS, Yates JR 3rd, Davis NG: Global analysis of protein palmitoylation in yeast. Cell 2006, 125:1003-1013.

66. Steinman RM, Banchereau J: Taking dendritic cells into medicine. Nature 2007, 449:419-426.

67. Aicart-Ramos C, Valero RA, Rodriguez-Crespo I: Protein palmitoylation and subcellular trafficking. Biochim Biophys Acta 1808, 2011:2981-2994.

68. Hernandez JL, Majmudar JD, Martin BR: Profiling and inhibiting reversible palmitoylation. Curr Opin Chem Biol 2013, 17:20-26.

69. Charron G, Tsou LK, Maguire W, Yount JS, Hang HC: Alkynyl-farnesol reporters for detection of protein S-prenylation in cells. Mol Biosyst 2011, 7:67-73.

70. Ingenuity pathway analysis software. www.ingenuity.com. 
71. Jiang Z, Georgel P, Li C, Choe J, Crozat K, Rutschmann S, Du X, Bigby T, Mudd S, Sovath S, Wilson IA, Olson A, Beutler B: Details of Toll-like receptor:adapter interaction revealed by germ-line mutagenesis. Proc Natl Acad Sci U S A 2006, 103:10961-10966.

72. Lopez CB, Yount JS, Hermesh T, Moran TM: Sendai virus infection induces efficient adaptive immunity independently of type I interferons. J Virol 2006, 80:4538-4545.

73. Yount JS, Hang HC: Proteins purified from DC2.4 cells labeled with DMSO or alk-16. PeptideAtlas 2014, http://www.peptideatlas.org/PASS/PASS00594.

74. Yount JS, Hang HC: Proteins purified from MEFs labeled with DMSO or alk-16. PeptideAtlas, 2014, http://www.peptideatlas.org/PASS/PASS00595.

doi:10.1186/s12915-014-0091-3

Cite this article as: Chesarino et al:: Chemoproteomics reveals Toll-like receptor fatty acylation. BMC Biology 2014 12:91.

\section{Submit your next manuscript to BioMed Central and take full advantage of:}

- Convenient online submission

- Thorough peer review

- No space constraints or color figure charges

- Immediate publication on acceptance

- Inclusion in PubMed, CAS, Scopus and Google Scholar

- Research which is freely available for redistribution 